\title{
miR-133b affects cell proliferation, invasion and chemosensitivity in renal cell carcinoma by inhibiting the ERK signaling pathway
}

\author{
YUAN XU ${ }^{1}$, YUAN MA ${ }^{2}$, XIAO-LING LIU ${ }^{1}$ and SHENG-LI GAO ${ }^{3}$ \\ ${ }^{1}$ Department of Traditional Chinese Medicine, Shandong Provincial Hospital Affiliated to Shandong University, \\ Jinan, Shandong 250021; ${ }^{2}$ Department of Internal Medicine, School of Medicine, Shandong University, Jinan, \\ Shandong 250100; ${ }^{3}$ Department of Clinical Medicine, Shandong Medical College, Jinan, Shandong 250021, P.R. China
}

Received June 4, 2019; Accepted February 11, 2020

DOI: $10.3892 / \mathrm{mmr} .2020 .11125$

\begin{abstract}
Renal cell carcinoma has the highest incidence rate of cancer types in the urinary system. Moreover, microRNAs (miRNA) have been closely associated with numerous types of tumor. The present study aimed to investigate the effects of miRNA (miR)-133b on the proliferation, invasion and chemosensitivity of renal cell carcinoma cells, and to determine whether its mechanism was regulated by the ERK signaling pathway. Both renal cell carcinoma and adjacent healthy tissues from 60 patients, in addition to renal cell carcinoma lines, ACHN, Caki-1, A-498 and 786-O, and 293 cells, were used in this study. miR-133b expression was measured from renal cell carcinoma, adjacent healthy tissues and renal cell carcinoma cell lines by reverse transcription-quantitative PCR. Cells were transfected with miR-133b mimic to achieve miR-133b overexpression. The proliferative, migratory and invasive ability of the cells were evaluated using MTT, wound healing and Matrigel assays, respectively, and flow cytometry was used to detect the apoptotic rate. Following treatment with an ERK inhibitor, U0126, and activator, LM22B-10, western blotting was used to detect the expression of related proteins and the activity of the ERK signaling pathway. The overexpression of miR-133b significantly inhibited cell proliferation, migration and invasion, whilst inducing apoptosis and increasing the drug sensitivity of renal cell carcinoma cells to cisplatin, docetaxel and doxorubicin. The miR-133b mimic also increased the protein expression levels of Bax and decreased the expression levels of matrix metalloproteinase (MMP)-2, MMP-9, ATP-binding cassette subfamily G2, P-glycoprotein, Bcl-2 and proliferating cell nuclear antigen, as well as the phosphorylation of ERK $(\mathrm{P}<0.05)$. The administration of the U0216 inhibitor demonstrated similar effects to miR-133b overexpression,
\end{abstract}

Correspondence to: Dr Sheng-Li Gao, Department of Clinical Medicine, Shandong Medical College, 5460 Second Ring South Road, Shizhong, Jinan, Shandong 250021, P.R. China

E-mail: guohuai8898249@126.com

Key words: microRNA-133b, renal cell carcinoma, proliferation, invasion, chemosensitivity, ERK signaling pathway and there was no significant difference compared with the miR-133b mimic transfection $(\mathrm{P}>0.05)$. However, the overexpression of miR-133b combined with LM22B-10 treatment weakened the anticancer effects of miR-133b mimic transfection $(\mathrm{P}<0.05)$. In conclusion, miR-133b overexpression was observed to inhibit the proliferation, migration and invasion of renal cell carcinoma cells and improve chemotherapeutic sensitivity; it was suggested that the mechanism maybe related to the inhibition of ERK1/2 phosphorylation and thus decreased ERK signaling pathway activity.

\section{Introduction}

Renal cell carcinoma is one of the most common types of kidney tumor originating from the renal tubular epithelium and has the highest incidence rate of cancer types found in the urinary system (1). According to cancer statistics in the United States, in 2018 there were 65,340 new cases of renal cell carcinoma, which accounted for $43.46 \%$ of the total number of urinary cancers diagnosed; of these cases, 14,970 resulted in death, accounting for $45.13 \%$ of the total number of urinary cancer deaths (2). Amongst adult malignant tumors, the incidence of renal cell carcinoma is $\sim 3 \%$ (1), and $\sim 30 \%$ of patients with renal cell carcinoma present with metastasis at the time of diagnosis (3). Surgical resection remains an effective treatment option for renal cell carcinoma, as the cancer cells are usually resistant to chemical drug treatment (4), which is the main contributing factor to the short survival time of patients. It has been discovered that certain factors are related to the tolerance of tumors to chemotherapeutic agents; for example, the regulation of drug uptake and elimination by renal cell carcinoma cells is mediated through membrane translocation-related proteins, such as P-glycoprotein (P-gp) and multidrug resistance-associated proteins (5).

MicroRNAs (miRNAs/miRs) are a class of non-coding RNAs that have no open reading frame in their sequences and therefore do not encode proteins (6). The abnormal expression of miRNAs has been closely associated with numerous types of tumour (7); they have been found to serve important roles in the development and progression of tumors, further to regulating cell migration, proliferation, differentiation and apoptosis by controlling the functions of oncogenes and tumor suppressor genes $(7,8)$. Of note, one study observed that multiple 
miRNAs are abnormally expressed in renal cell carcinoma (9), whilst another study found that miRNAs were highly stable in the serum, easy to detect and not easily degraded (10). These findings provided a theoretical and methodological basis for studying the function of miRNAs as biomarkers of renal cell carcinoma. In fact, one study suggested that miR-133b may be used as a tumor suppressor gene to regulate cell growth in types of cancer $(11,12)$. For example, the expression levels of miR-133b were found to be increased in lung cancer, which prevented lung cancer cells from proliferating, whilst promoting cell apoptosis (11). Similarly, a previous study demonstrated that miR-133b can inhibit the proliferation, migration and invasion of esophageal cancer cells (12).

The ERKs, including ERK1 and ERK2, are involved in the transmission of extracellular signals intracellularly (13). Upon activation by phosphorylation, theERK protein translocates into the nucleus from the cytoplasm, where it transmits signals into the nucleus to participate in various biological reactions (13). The ERK protein is considered to be the convergence point of multiple signaling pathways that are involved in various biological functions, such as cell development, colonization, apoptosis and malignant transformation, amongst others (13). One previous study reported that miR-133b shortened the latency of cervical cancer and promoted the occurrence and metastasis through activating the ERK and AKT signaling pathways in mice (14); however, to the best of our knowledge, there are no studies on the relationship between miR-133b and ERK in renal cell carcinoma. In addition, the ERK signaling pathway is closely associated with the chemosensitivity of cells, for instance, the inactivation of the ERK signaling pathway was observed to increase the chemosensitivity of osteosarcoma U2OS cells to cisplatin (15). Thus, in the present study, the expression levels of miR-133b in renal cell carcinoma were determined and its effect on cell proliferation, invasion and chemosensitivity, further its potential mechanisms were investigated.

\section{Materials and methods}

Patient studies. The present study was approved by the Research Ethics Committee of School of Medicine, Shandong University and was conducted under the Declaration of Helsinki principles. Informed written consent was obtained from all participating patients. A total of 60 patients with renal cell carcinoma (43 male patients; 17 female patients; age, 27-73 years; mean age, 55.14 \pm 10.45 years) at the Shandong Provincial Hospital Affiliated to Shandong University were recruited from June 2017 to July 2018. All patients had comprehensive clinical pathological data and did not receive chemotherapy, radiation therapy or immunotherapy before surgery. The patients' renal cell carcinoma and adjacent healthy tissue specimens were collected and the specimens were subsequently stored in liquid nitrogen or $4 \%$ paraformaldehyde solution within $10 \mathrm{~min}$ of being removed, then stored at $-80^{\circ} \mathrm{C}$. Samples were confirmed to be either renal cell carcinoma tissue or healthy tissue by histopathological diagnosis.

Cell collection, culture and treatment. In total, four human renal cell carcinoma cell lines, ACHN, Caki-1, A-498 and 786-O, and the 293 cell line were obtained from the American
Type Culture Collection. In further experiments, 786-O cells were used. All cells were cultured in RPMI-1640 medium (Gibco; Thermo Fisher Scientific, Inc.) supplemented with 10\% FBS (Sigma-Aldrich; Merck KGaA), $100 \mathrm{U} / \mathrm{ml}$ penicillin and $100 \mathrm{mg} / \mathrm{ml}$ streptomycin, and maintained in a $5 \% \mathrm{CO}_{2}$ incubator (Thermo Fisher Scientific, Inc.) at $37^{\circ} \mathrm{C}$.

The 786-O cells were collected for experiments upon reaching the logarithmic growth phase and were divided into 23 groups: i) The control group, which contained untreated cells; ii) the negative control (NC) group, which contained cells transfected with the miR-133b mimic NC; iii) the miR-133b mimic (miR-133b) group, which contained cells transfected with the miR-133b mimic; iv-viii) the (DDP; MedChemExpress) groups, which received $0,2.5,5,10$ or $20 \mu \mathrm{g} / \mathrm{ml}$ DDP; ix-xiii) the docetaxel (DXT; MedChemExpress) groups, which received $0,5,10,20$ or $40 \mu \mathrm{g} / \mathrm{ml} \mathrm{DXT}$; xiv-Xviii) the doxorubicin (ADR; MedChemExpress) groups, which received $0,0.25,0.5,1$ or $2 \mu \mathrm{g} / \mathrm{ml} \mathrm{DXT}$; xix) the miR-133b + DDP group, which contained cells transfected with the miR-133b mimic and treated with $5 \mu \mathrm{g} / \mathrm{ml}$ DDP; $\mathrm{xx}$ ) the miR-133b + DXT group, which contained cells transfected with miR-155p mimic and treated with $20 \mu \mathrm{g} / \mathrm{ml}$ DXT; xxi) the miR-133b + ADR group, which contained cells transfected with miR-133b mimic and treated with $0.5 \mu \mathrm{g} / \mathrm{ml}$ ADR; xxii) the U0126 group, which contained cells treated with the ERK pathway signal transduction inhibitor, U0216 (20 $\mu \mathrm{mol} /$; Selleck Chemicals); and xxiii) the miR-133b + LM22B-10 group, which contained cells transfected with the miR-133b mimic and treated with the ERK activator LM22B-10 (5 mmol/l; cat. no. HY-104047; MedChemExpress).

Cell transfection. The 786-O cells were digested and passaged with $0.25 \%$ trypsin (Invitrogen; Thermo Fisher Scientific, Inc.), and $2 \times 10^{5}$ cells in the exponential growth phase were plated into 6 -well plates. Following $24 \mathrm{~h}$ of incubation at $37^{\circ} \mathrm{C}$ with $5 \% \mathrm{CO}_{2}$, cell growth was observed using an inverted fluorescent microscope (Olympus Corporation) under x200 magnification. Upon reaching 30-50\% confluence, the miR-133b mimic (5'-UUUGGUCCCCUUCAACCA GCUA-3') and the miR-133b mimic NC (5'-UUUGGUAAA AUUCAACCAGCUA-3'; Guangzhou RiboBio Co., Ltd.) were transfected into the cells at a concentration of $20 \mathrm{nmol} / \mathrm{l}$ to construct transiently overexpressing miR-133b cell lines using Lipofectamine ${ }^{\circledR} 2000$ reagent (Invitrogen; Thermo Fisher Scientific, Inc.), according to the manufacturer's protocol. After $24 \mathrm{~h}$, the proof of successful transfection was analyzed using reverse transcription-quantitative PCR (RT-qPCR).

$R T-q P C R$. Total RNA was extracted from cells using the MagMAX ${ }^{\mathrm{TM}}$ mirVana ${ }^{\mathrm{TM}}$ Total RNA Isolation kit (cat. no. A27828; Thermo Fisher Scientific, Inc.) according to the manufacturer's protocol. Total RNA was reverse transcribed into cDNA at $42^{\circ} \mathrm{C}$ for $1 \mathrm{~h}$ then $90^{\circ} \mathrm{C}$ for $5 \mathrm{~min}$ with a high-capacity RNA to cDNA ${ }^{\mathrm{TM}}$ kit (cat. no. 4387406; Thermo Fisher Scientific, Inc.). qPCR was subsequently performed using SYBR-Green qPCR Master Mix (MedChemExpress) and $2 \mu \mathrm{l}$ cDNA as a template. The following primer pairs (Guangzhou RiboBio Co., Ltd.) were used for the qPCR: miR-133b forward, 5'-AAAGGACCCCAACAACCAGCA A-3' and reverse, 5'-TTGCTGGTTGTTGGGGTCCTTT-3'; 
and U6 forward, 5'-CTCGCTTCGGCAGCACATATACT-3' and reverse, 5'-ACGCTTCACGAATTTGCGTGTC-3'. The following thermocycling conditions were used for the PCR: Initial denaturation at $95^{\circ} \mathrm{C}$ for $2 \mathrm{~min}$; and 40 cycles of $95^{\circ} \mathrm{C}$ for $15 \mathrm{sec}$ and $60^{\circ} \mathrm{C}$ for $1 \mathrm{~min}$. Expression levels of miR-133b were quantified using the $2^{-\Delta \Delta C q}$ method (16) and normalized to the internal reference gene U6 gene.

MTT assay for cell proliferation. The 786-O cells were digested using trypsinase and $1 \times 10^{4}$ cells $/ \mathrm{ml}$ were plated into 96-well culture plates and incubated with $5 \% \mathrm{CO}_{2}$ at $37^{\circ} \mathrm{C}$. At 24, 48 and $72 \mathrm{~h}, 20 \mu \mathrm{l}$ MTT reagent $(5 \mathrm{mg} / \mathrm{ml})$ was added and then cells were further cultured for $4 \mathrm{~h}$ in a $37^{\circ} \mathrm{C}$ incubator. The medium was discarded and $200 \mu 1$ DMSO (Sigma-Aldrich; Merck KGaA) was added. The absorbance [optical density (OD) value] of each well was measured at a wavelength of $490 \mathrm{~nm}$ using a spectrophotometer (Bio-Rad Laboratories, Inc.).

Wound healing assay. The 786-O cells in the exponential growth phase were digested with $0.25 \%$ trypsin into a single cell suspension and adjusted to a concentration of $3 \times 10^{5}$ cells $/ \mathrm{ml}$. A total of $1 \mathrm{ml}$ cell suspension was added to each well of the 6-well plate. Upon cells reaching confluence, a 10- $\mu$ l pipette tip was used to create a single scratch on the cell monolayer in a sterile environment. Cells were then washed with PBS to remove the cell debris. Subsequently, RPMI-1640 medium was added and cells were incubated at $37^{\circ} \mathrm{C}$ and $5 \% \mathrm{CO}_{2}$. Cells were visualized using an inverted fluorescent microscope (Olympus Corporation) and the wells were imaged at 0 and $24 \mathrm{~h}$. The degree of wound healing was analyzed by ImageJ software (version 6.0, National Institutes of Health).

Matrigel assay. A total of $2 \times 10^{5} 786-\mathrm{O}$ cells $/ \mathrm{ml}$ were collected with $0.25 \%$ trypsin-EDTA solution and plated in the upper chambers of a Transwell plate in $100 \mu 1$ RPMI-1640 DMEM (Gibco; Thermo Fisher Scientific, Inc.). Transwell membranes were precoated with $50 \mu \mathrm{l} \mathrm{Matrigel} \mathrm{for} 30 \mathrm{~min}$ at $37^{\circ} \mathrm{C}$. A total of $600 \mu 1$ complete RPMI-1640 medium supplemented with $10 \%$ FBS was plated in the lower chambers. Following incubation for $24 \mathrm{~h}$ at $37^{\circ} \mathrm{C}$, the invasive cells were fixed in $4 \%$ paraformaldehyde for $30 \mathrm{~min}$ at $37^{\circ} \mathrm{C}$ and then stained with $1 \%$ crystal violet (cat. no. G1062; Beijing Solarbio Science \& Technology Co., Ltd.) for $20 \mathrm{~min}$ at $37^{\circ} \mathrm{C}$. Stained cells were counted in nine randomly selected fields using an optical fluorescent microscope under x400 magnification (Olympus Corporation). The number of invasive cells was subsequently calculated by ImageJ (version 6.0; National Institutes of Health).

Flow cytometric analysis of apoptosis. The 786-Ocells in the logarithmic phase were trypsinized and were collected by centrifugation at $999 \mathrm{x} \mathrm{g}$ for $5 \mathrm{~min}$ at $4^{\circ} \mathrm{C}$. Cells were subsequently thoroughly washed twice with pre-cooled sterile PBS at $4^{\circ} \mathrm{C}$. A total of $1 \times 10^{6}$ cells $/ \mathrm{ml}$ were resuspended in $250 \mu \mathrm{l}$ $1 \mathrm{X}$ binding buffer, of which $195 \mu \mathrm{l}$ was subsequently added to $5 \mu \mathrm{l}$ Annexin V-FITC and mixed for $3 \mathrm{~min}$. Then, $10 \mu \mathrm{l}$ propidium iodide (PI) solution $(20 \mu \mathrm{g} / \mathrm{ml})$ was added and mixed. Following incubation for $10 \mathrm{~min}$ at room temperature in the dark, $1 \mathrm{X}$ binding buffer $(400 \mu \mathrm{l})$ was added and gently mixed. Apoptotic cells were subsequently analyzed using a Gallios flow cytometer (Beckman Coulter, Inc.) and BD CellQuest ${ }^{\mathrm{TM}}$ software (version 5.1; BD Biosciences). The Q4 data reflected the apoptotic rate in this study.

Detection of cellular activity using the MTS assay. The 786-O cells in the logarithmic growth phase were digested with $0.25 \%$ trypsin and seeded into 96 -well plates at a cell density of $3 \times 10^{5}$ cells $/ \mathrm{ml}$. Following treatment for $48 \mathrm{~h}$ at $37^{\circ} \mathrm{C}, 20 \mu 1 \mathrm{MTS}$ reagent (Promega Corporation) was added to each well and incubated at $37^{\circ} \mathrm{C}$ for $3 \mathrm{~h}$. A microplate reader was used to record the OD value at $490 \mathrm{~nm}$. The cells that received treatments were set as the experimental groups and $0 \mu \mathrm{g} / \mathrm{ml}$ drug with same volume saline treatmentwas used as the control group. Distilled water was used as the blank group. The cell survival rate (\%) was calculated using the following formula: (OD490 experimental group $\left.-\mathrm{OD} 490_{\text {blank group }}\right) /\left(\mathrm{OD} 490_{\text {control }}\right.$ group $\left.-\mathrm{OD} 490_{\text {blank group }}\right) \mathrm{x} 100$. The experiment was repeated in triplicate.

Detection of chemosensitivity using the MTT assay. The 786-O cells were digested by trypsinase, and a total of $1 \times 10^{4} / \mathrm{ml}$ cells were subsequently plated into 96 -well plates and incubated as described above. Different doses of DDP, DXT and ADR were administrated to evaluate the IC50 of each drug. Following $48 \mathrm{~h}$ of culture, $20 \mu 15 \mathrm{mg} / \mathrm{ml}$ MTT was added to each well and incubated at $37^{\circ} \mathrm{C}$ for $4 \mathrm{~h}$. The IC50 was analyzed by SPSS 19.0 (SPSS, Inc.). After adherence, the miR-133b groups were treated with $5 \mu \mathrm{g} / \mathrm{ml}$ DDP, $20 \mu \mathrm{g} / \mathrm{ml}$ DXT or $0.5 \mu \mathrm{g} / \mathrm{ml}$ ADR, respectively. Following 24, 48 and $72 \mathrm{~h}$ of culture, $20 \mu 15 \mathrm{mg} / \mathrm{ml}$ MTT was added to each well of all groups and incubated at $37^{\circ} \mathrm{C}$ for $4 \mathrm{~h}$. The supernatant was discarded and $200 \mu 1$ DMSO (Sigma-Aldrich; Merck KGaA) was added. The OD value was measured at $490 \mathrm{~nm}$ using a spectrophotometer (Bio-Rad Laboratories, Inc.). The proliferative ability of the cells in each group following treatment with the different drugs was evaluated to determine the effect of the overexpression of miR-133b on drug chemosensitivity.

Western blotting. The expression levels of proliferating cell nuclear antigen (PCNA), matrix metalloproteinase (MMP)-2, MMP-9, Bcl-2, Bax, ATP-binding cassette subfamily G2 (ABCG2), P-gp, phosphorylated (p)-ERK1/2 and ERK1/2 were analyzed using western blotting. Total protein was extracted from 786-O cells with a total protein extraction kit (cat. no. BC3640-50T; Beijing Solarbio Science \& Technology Co., Ltd.), according to the manufacturer's protocol. Total protein was quantified using a bicinchoninic acid assay kit (cat. no. 23225; Pierce; Thermo Fisher Scientific, Inc.) and $40 \mu \mathrm{g}$ protein/lane was separated via $10 \%$ SDS-PAGE (Bio-Rad Laboratories, Inc.). The separated proteins were subsequently transferred onto a PVDF membrane (EMD Millipore) and blocked with $5 \%$ skim milk at $25^{\circ} \mathrm{C}$ for $1 \mathrm{~h}$. The membranes were incubated overnight at $4{ }^{\circ} \mathrm{C}$ with the following primary antibodies diluted in 5\% BSA (Sigma-Aldrich; Merck KGaA): Anti-MMP-2 (1:1,000; cat. no. ab37150; Abcam); anti-MMP-9 $(1: 1,000$; cat. no. ab38898; Abcam); anti-p-ERK1/2 $(1: 1,000$; cat. no. ab176640; Abcam); anti-ERK1/2 (1:1,000; cat. no. ab17942; Abcam); anti-PCNA (1:1,000; cat. no. ab152112; Abcam); anti-Bax (1:1,000; cat. no. ab53154; Abcam); anti-Bcl-2 (1:1,000; cat. no. ab59348; Abcam); 

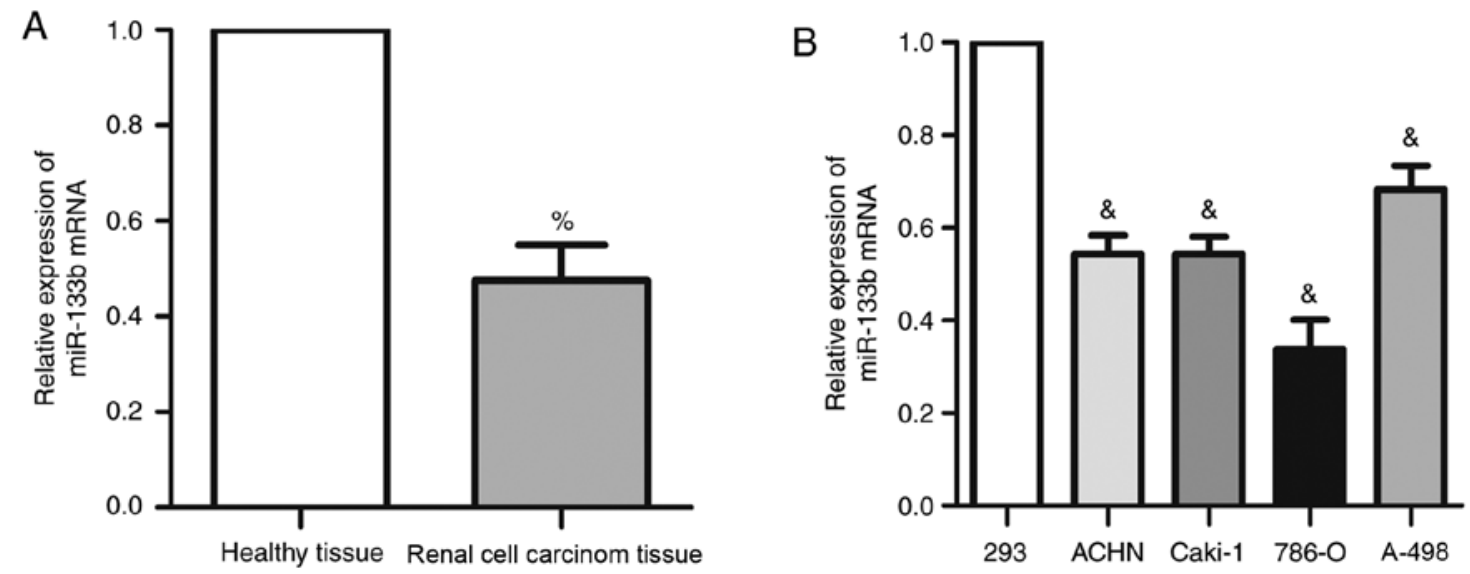

Figure 1. miR-133b expression levels are decreased in renal cell carcinoma tissues and cells. Reverse transcription-quantitative PCR was used to determine the expression levels of miR-133b in (A) renal cell carcinoma tissues and (B) renal cell carcinoma cell lines. ${ }^{\%} \mathrm{P}<0.05$ vs. healthy tissue; ${ }^{\&} \mathrm{P}<0.05$ vs. 293. miR, microRNA.

anti-ABCG2 (1:1,000; cat. no. ab63907; Abcam); anti-P-gp (1:1,000; cat. no. ab129450; Abcam); and anti- $\beta$-actin $(1: 1,000$; cat. no. ab8227; Abcam). Following the primary antibody incubation, the membranes were washed thrice with TBS- $0.01 \%$ Tween-20 (TBST) for 10 min each and then incubated with a horseradish peroxidase-conjugated goat anti-rabbit IgG secondary antibody (1:2,000; cat. no. ab6721; Abcam) for $1 \mathrm{~h}$ at room temperature. The membranes were washed thrice with TBST. Protein bands were visualized using an ECL chemiluminescence reagent (Hanbio Biotechnology Co., Ltd.). Protein expression was quantified using ImageJ software (version 6; National Institutes of Health) and normalized to $\beta$-actin.

Statistical analysis. Statistical analysis was performed using SPSS version 19.0 software (IBM Corp.). The experiments were repeated three times and data are expressed as the mean \pm SD. Statistical differences between two groups were determined using a Student's t-test, whereas the statistical differences between multiple groups were analyzed using a one-way ANOVA followed by Tukey's test for multiple comparisons. $\mathrm{P}<0.05$ was considered to indicate a statistically significant difference.

\section{Results}

miR-133b expression is decreased in renal cell carcinoma tissues and cells. The expression levels of miR-133b were detected using RT-qPCR in 60 cases of renal cell carcinoma and adjacent healthy tissues. The expression levels of endogenous miR-133b in renal cell carcinoma were significantly decreased compared with the adjacent healthy tissues (Fig. 1A). Similarly, miR-133b expression was significantly decreased in all four renal cell carcinoma cell lines compared with 293 cells, with the most significant difference being observed in 786-O cells $(\mathrm{P}<0.05)$. Therefore, 786-O cells were used for subsequent experiments. These findings suggested that miR-133b expression may be decreased in both renal cell carcinoma tissues and renal carcinoma cell lines.

Overexpression of miR-133b inhibits the proliferation and invasion of renal cell carcinoma cells. The efficiency of
miR-133b transfection in 786-O cells was quantified using RT-qPCR (Fig. 2A); there was no significant difference in the relative expression levels of miR-133b between the control group and the NC group ( $\mathrm{P}>0.05)$, whereas the expression levels of miR-133b mRNA were significantly increased in the miR-133b group when compared to $\mathrm{NC}$ group $(\mathrm{P}<0.05)$.

Following 24, 48 and $72 \mathrm{~h}$ of cell culture, an MTT assay was used to analyze the proliferation of cells in each group (Fig. 2B). Compared with the control group, there was no significant difference observed in the proliferative rate in the $\mathrm{NC}$ group $(\mathrm{P}>0.05)$; however, there was significant difference in the proliferative rate of the miR-133b group $(\mathrm{P}<0.05)$, in which the proliferative rate of the cells in the miR-133b group was significantly reduced compared with cells in the $\mathrm{NC}$ group. These findings suggested that the overexpression of miR-133b may reduce the proliferative ability of renal carcinoma cells.

A wound healing and Matrigel assay were used to determine the migratory and invasive ability, respectively, of 786-O cells (Fig. 2C and D). The wound healing rate and the number of invasive cells in the miR-133b group were significantly reduced compared with the control and $\mathrm{NC}$ groups $(\mathrm{P}<0.05)$, which indicated that the overexpression of miR-133b may inhibit the migratory and invasive ability of renal carcinoma cells.

The expression levels of PCNA, MMP-2 and MMP-9 were analyzed using western blotting (Fig. 2E). The expression levels of PCNA, MMP-2 and MMP-9 in the miR-133b group were significantly reduced compared with the control and $\mathrm{NC}$ groups $(\mathrm{P}<0.05)$. These results suggested that the overexpression of miR-133b may inhibit the expression of proliferation and invasion-related proteins.

Overexpression of miR-133b induces cells apoptosis and enhances drug sensitivity. The apoptotic rate of renal carcinoma cells was detected using flow cytometry (Fig. 3A). Compared with the control and NC groups, the apoptotic rate of the miR-133b group was significantly increased $(\mathrm{P}<0.05)$, suggesting that the overexpression of miR-133b may promote the apoptosis of renal cell carcinoma cells.

Cellular viability was subsequently analyzed using a MTT assay; following treatment with a range of doses of DDP, DXT and ADR for $48 \mathrm{~h}$, the cell activity was observed to decrease 
A

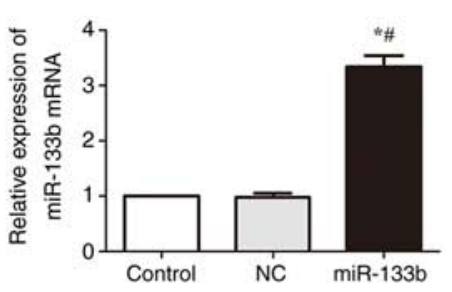

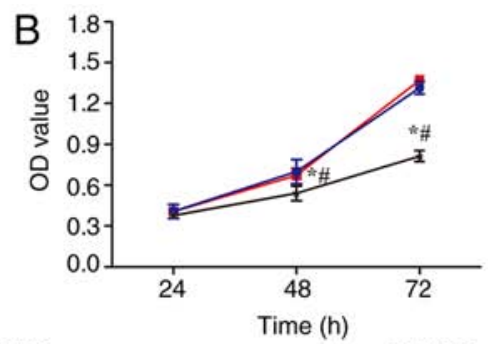

NC

$$
\begin{aligned}
& \text { - Control } \\
& \text { - NC } \\
& \text { - miR-133b }
\end{aligned}
$$

C
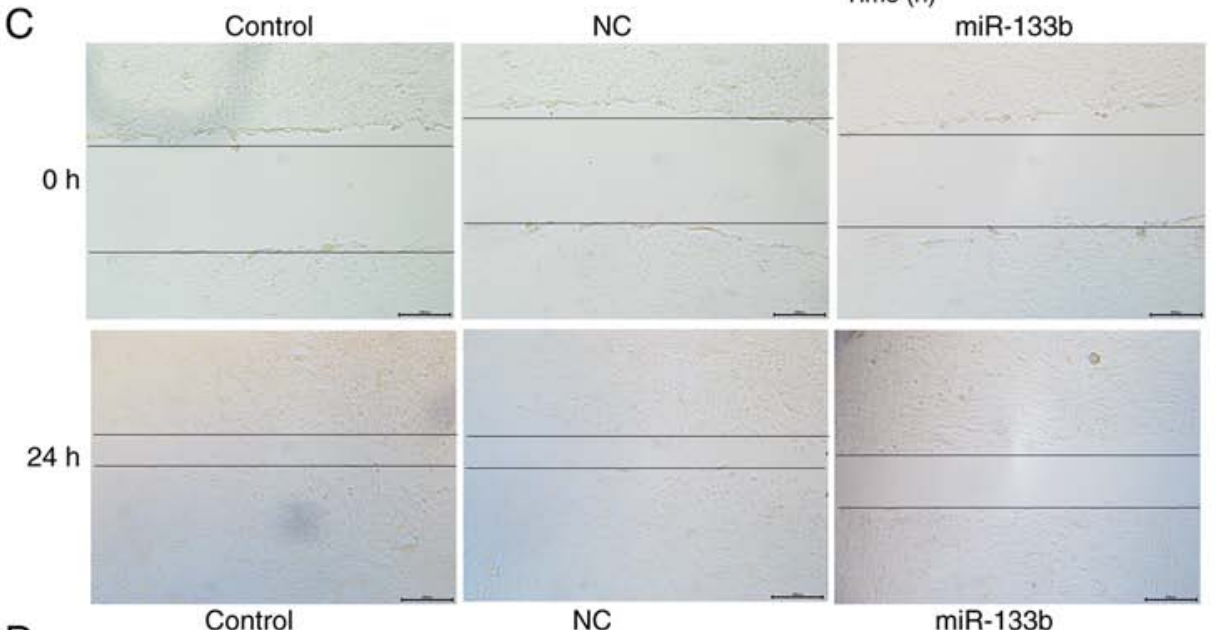

D
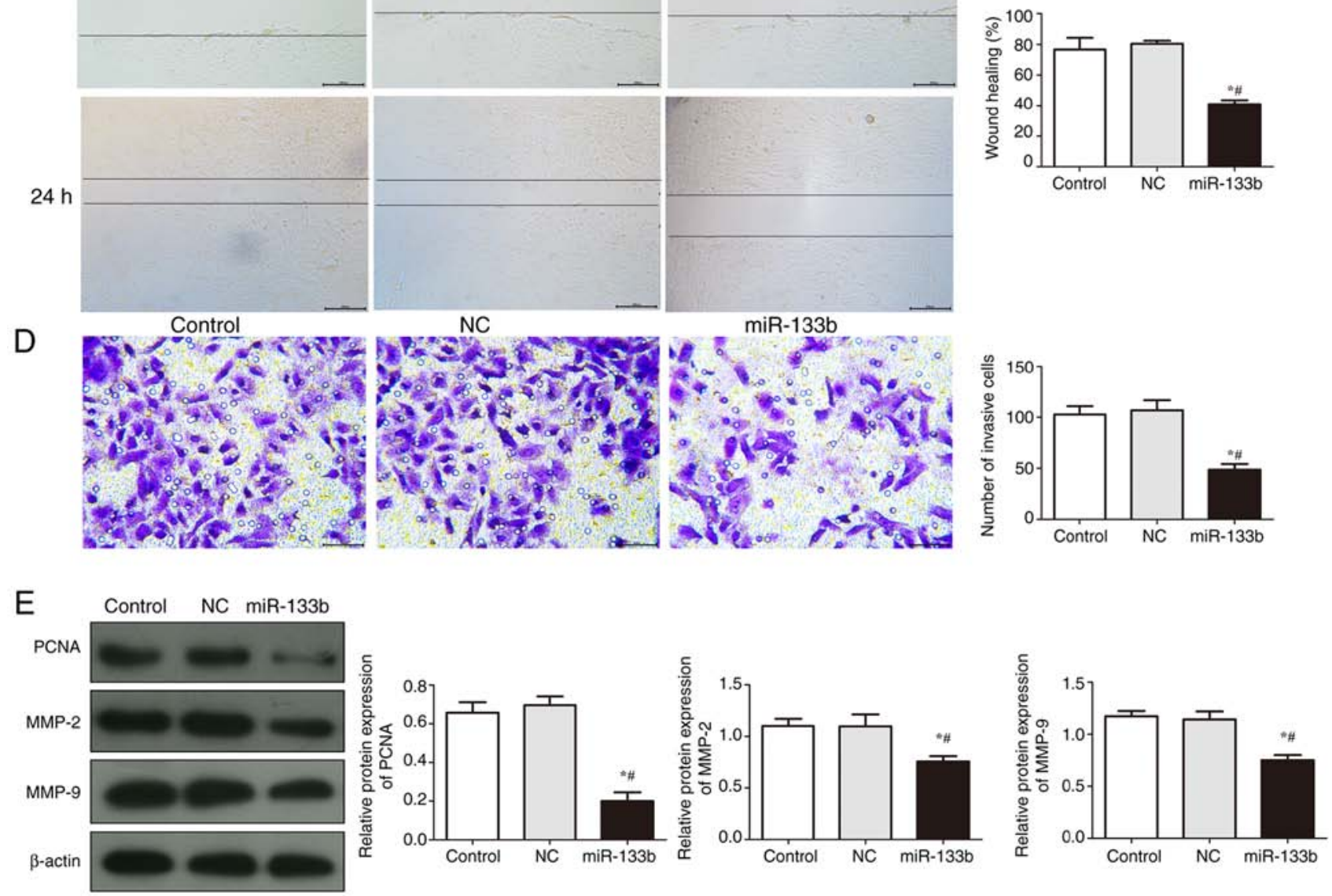

Figure 2. Overexpression of miR-133b inhibits proliferation and invasion in 786-O cells. (A) Transfection efficiency of miR-133 was determined using reverse transcription-quantitative PCR. (B) Cell proliferation was detected using an MTT assay following miR-133b overexpression. (C) Wound healing assay was used to investigate cell migration following miR-133b overexpression, scale bar, $200 \mu \mathrm{m}$. (D) Matrigel assay was used to investigate the number of invasive cells following miR-133b overexpression, scale bar, $50 \mu \mathrm{m}$. (E) Western blotting was used to analyze the expression levels of PCNA, MMP-2 and MMP-9 following miR-133b overexpression. " $\mathrm{P}<0.05$ vs. control; ${ }^{*} \mathrm{P}<0.05$ vs. NC. miR, microRNA; NC, negative control; PCNA, proliferating cell nuclear antigen; MMP, matrix metalloproteinase; OD, optical density.

in a dose-dependent manner with all three drugs (Fig. 3B). Drug concentration values close to each drug's half-maximal inhibitory concentration value (DDP, $5 \mu \mathrm{g} / \mathrm{ml}$; DXT, $20 \mu \mathrm{g} / \mathrm{ml}$; ADR, $0.5 \mu \mathrm{g} / \mathrm{ml}$ ) were used in the subsequent experiments.

The effects of overexpressing miR-133b on the drug sensitivity of 786-O cells to DDP, DXT and ADR were subsequently investigated using an MTT assay (Fig. 3C). The cell proliferative ability of the miR-133b, DDP, DXT, ADR and the miR-133b + DDP/DXT/ADR groups was significantly decreased compared with the control and NC groups, and this effect was found to be time-dependent $(\mathrm{P}<0.05$; Fig. $3 \mathrm{C})$. The proliferative ability of the cells in the miR-133b + DDP/DXT/ADR group was inhibited to a greater level compared with the miR-133b, DDP/DXT/ADR groups, respectively. These results suggested that the overexpression of miR-133b may increase the sensitivity of renal carcinoma cells to these three drugs.

The expression levels of Bcl-2, Bax, ABCG2 and P-gp in each group were detected using western blotting (Fig. 3D and E). Compared with the control and NC groups, the protein expression levels of Bax in the miR-133b group were significantly increased, whilst the protein expression levels of ABCG2, P-gp and Bcl-2 were significantly decreased (Fig. 3D and E). These findings indicated that the overexpression of miR-133b may decrease the expression levels of drug resistance-related 
A
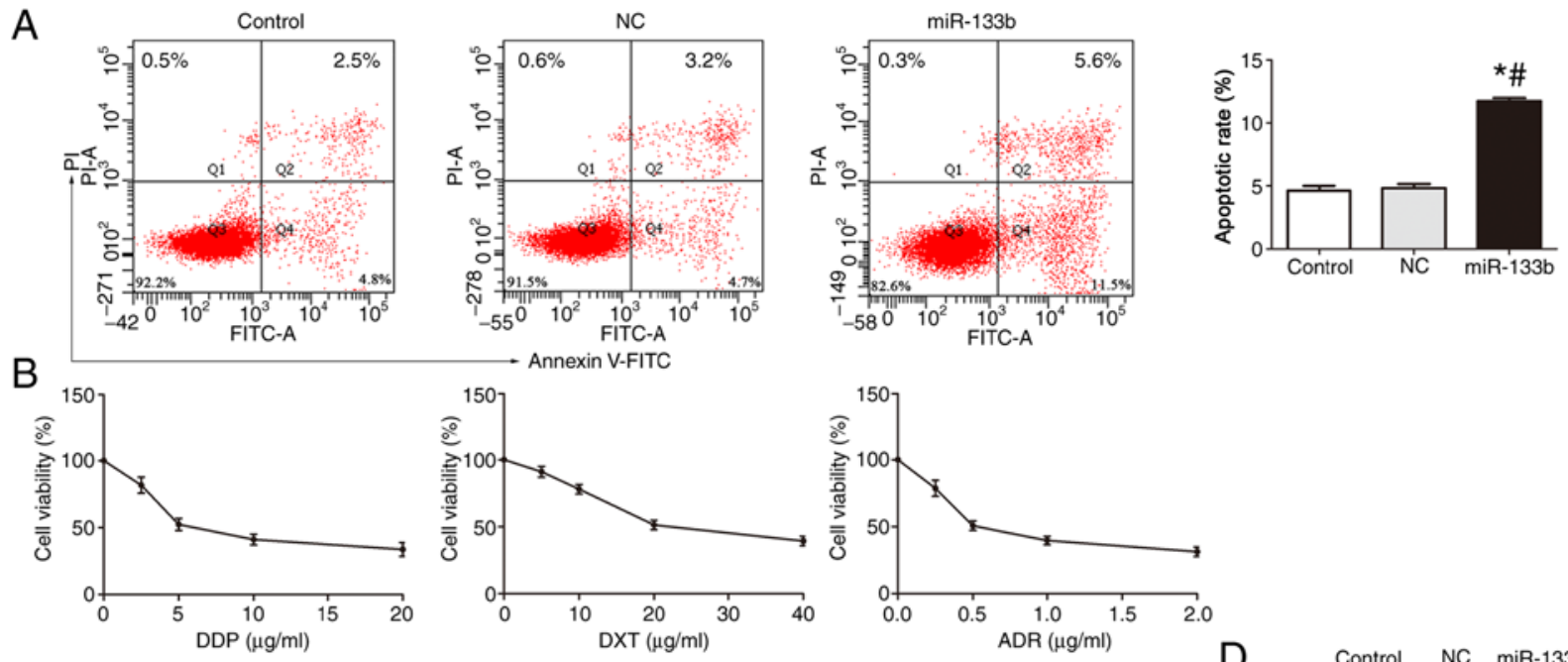

C
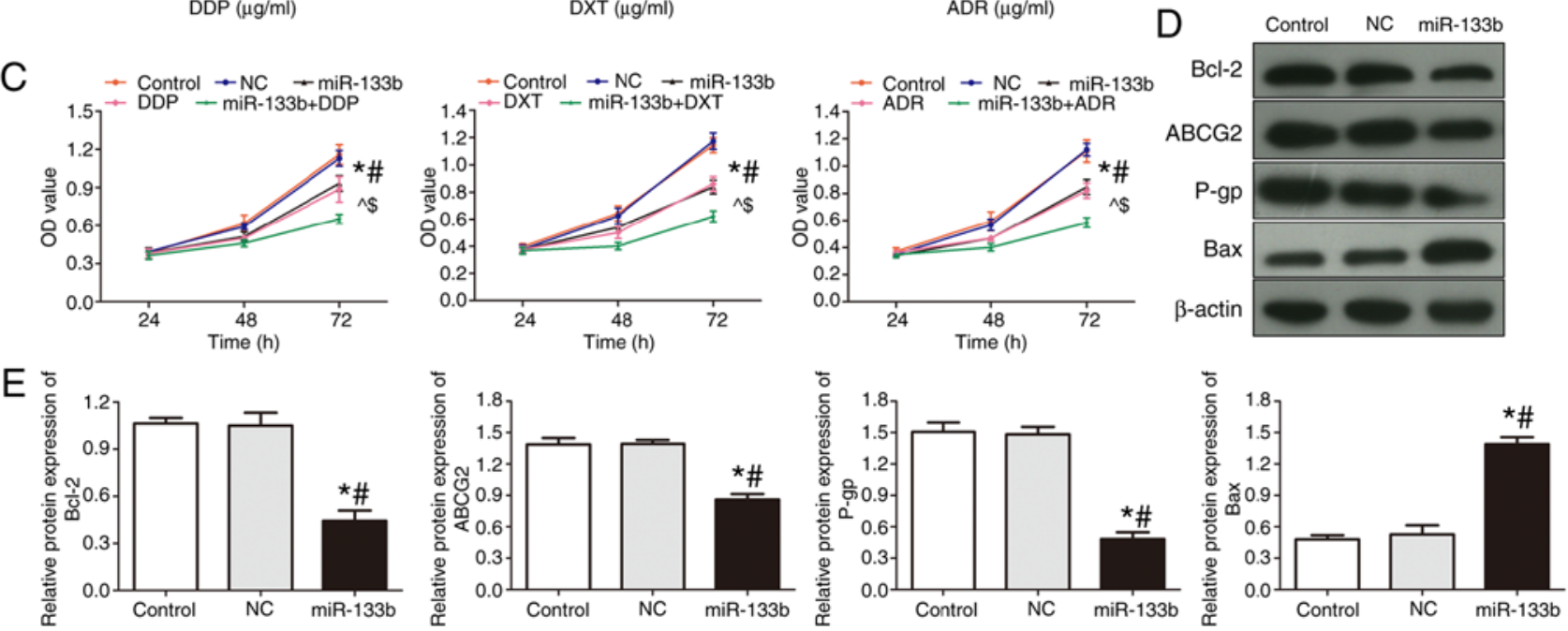

Figure 3. Overexpression of miR-133b induces apoptosis of 786-O cells and enhances drug sensitivity. (A) Flow cytometric analysis of apoptosis was performed following miR-133b overexpression. (B) MTS assay was used to investigate cell viability following the treatment of cells with increasing concentrations of DDP, DXT and ADR. (C) Proliferation of 786-O cells was investigated following the treatment of DDP, DXT or ADR and/or miR-133b overexpression. (D) Western blotting was used to investigate the protein expression levels of Bcl-2, Bax, ABCG2 and P-gp in cells following miR-133b overexpression. (E) Semi-quantitative analysis of protein expression levels. ${ }^{*} \mathrm{P}<0.05$ vs. control; ${ }^{\#} \mathrm{P}<0.05 \mathrm{vs}$. NC; ${ }^{\wedge} \mathrm{P}<0.05$ vs. miR-133b; ${ }^{\$} \mathrm{P}<0.05 \mathrm{vs}$. DDP/DXT/ADR. miR, microRNA; DDP, cisplatin; DXT, docetaxel; ADR, doxorubicin; ABCG2, ATP-binding cassette subfamily G2; P-gp, P-glycoprotein; NC, negative control; PI, propidium iodide; OD, optical density.

proteins ABCG2 and P-gp, and the anti-apoptotic protein Bcl-2, whilst increasing the levels of Bax.

Overexpression of miR-133b inhibits the activity of the ERK signaling pathway. The expression levels of p-ERK1/2 and ERK1/2 in each group were analyzed (Fig. 4). Compared with the control and NC groups, the protein expression levels of p-ERK1/2 in the miR-133b group were significantly decreased, whilst the protein expression levels of total ERK1/2 were not significantly different. Overall, the data suggested that the overexpression of miR-133b may inhibit ERK1/2 phosphorylation levels and reduce the activity of the ERK signaling pathway.

Effects of the miR-133b-mediated ERK signaling pathway on the occurrence and development of renal cell carcinoma. To further investigate whether miR-133b has a role in the occurrence and development of renal cell carcinoma through the ERK signaling pathway, the ERK pathway signal transduction inhibitor, U0126, was used, in addition to the signaling pathway inhibitor, LM22B-10, which was used in cells also overexpressing miR-133b. Compared with the NC group, the proliferative, migratory and invasive abilities of cells in the miR-133b and U0126 group were significantly decreased $(\mathrm{P}<0.05$, Fig. 5A-C). Compared with miR-133b group, the proliferative, migratory and invasive abilities of cells were significantly increased in miR-133b + LM22B-10 group ( $\mathrm{P}<0.05$; Fig. 5A-C). The protein expression levels of PCNA, MMP-2 and MMP-9 in the miR-133b and U0126 group were significantly decreased contrasted to control group, were decreased in miR-133b + LM22B-10 group compared with the miR-133b group $(\mathrm{P}<0.05$; Fig. 5D). The apoptotic rate and the sensitivity of 786-O cells to DDP, DXT and ADR were significantly increased in the miR-133b and U0126 group compared with miR-133b + LM22B-10 group ( $<<0.05$; Fig. 6A and B). Compared with miR-133b group and U0126 group, the expression levels of Bcl-2, ABCG2 and P-gp protein were significantly increased (Fig. 6C and D). Furthermore, the phosphorylation of ERK1/2 in different groups was observed (Fig. 7). Compared with NC group, the expression of p-ERK1/2/ERK1/2 was significantly decreased in miR-133b and U0126 group $(\mathrm{P}<0.05)$. 

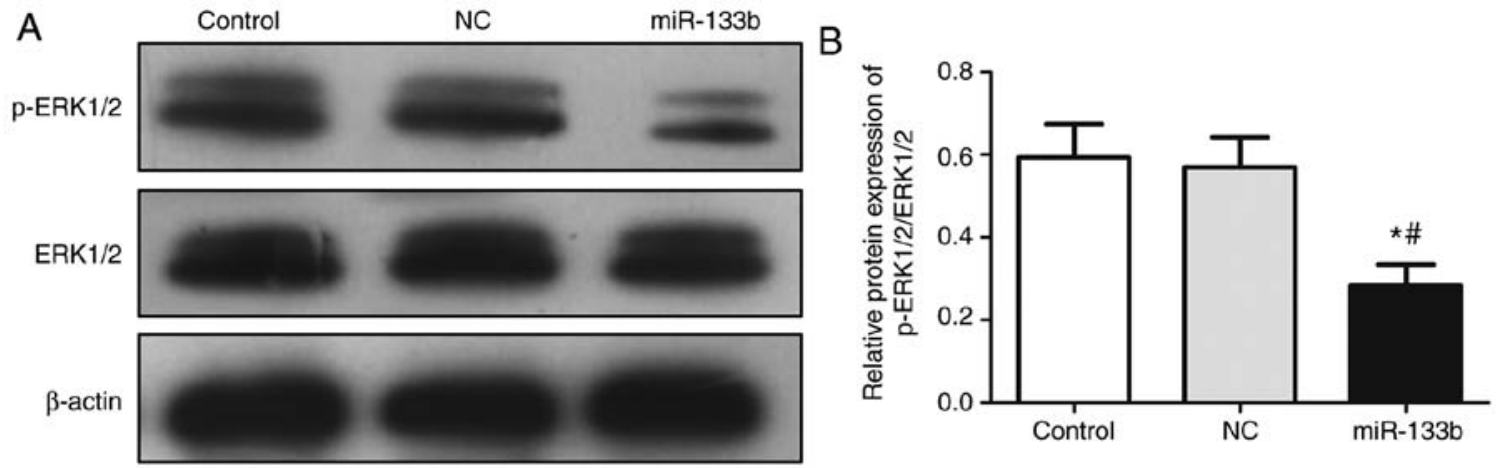

Figure 4. Effects of the overexpression of miR-133b on ERK signaling pathway activity. (A) Western blotting was performed to investigate the protein expression levels of $\mathrm{p}$-ERK1/2 and ERK1/2. (B) Semi-quantification of the expression levels. " $\mathrm{P}<0.05$ vs. control; ${ }^{~} \mathrm{P}<0.05$ vs. NC. miR, microRNA; $\mathrm{p}$, phosphorylated; NC, negative control.

A

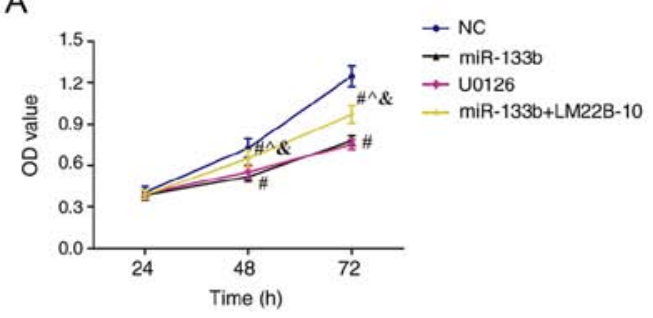

C

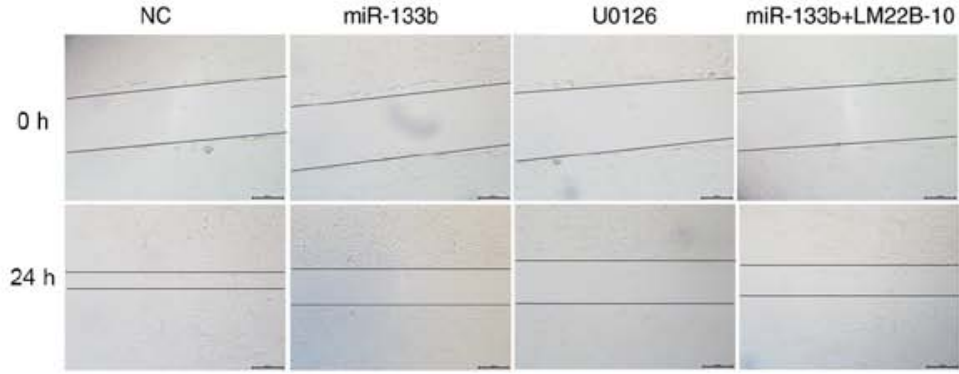

B
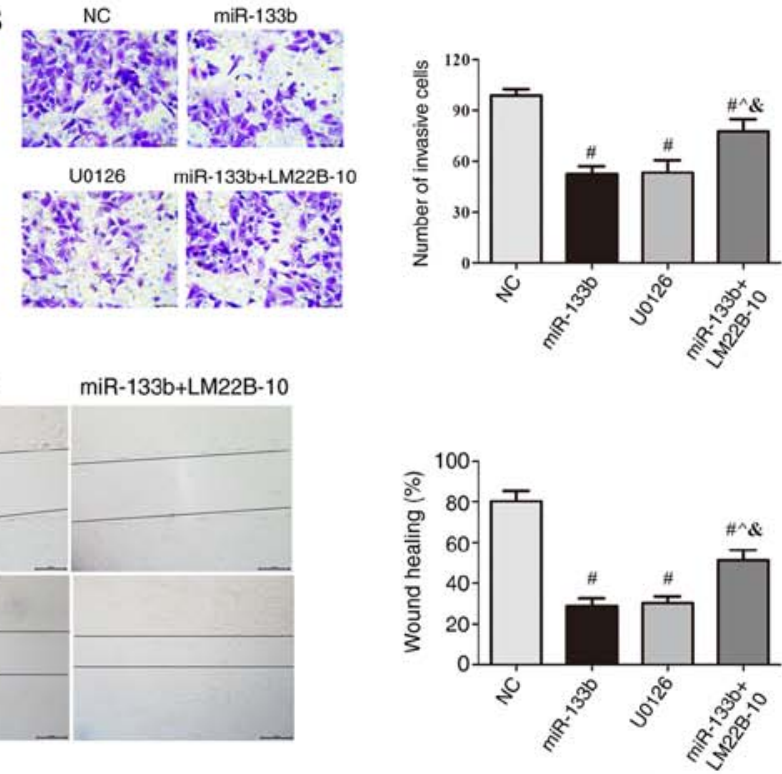

D
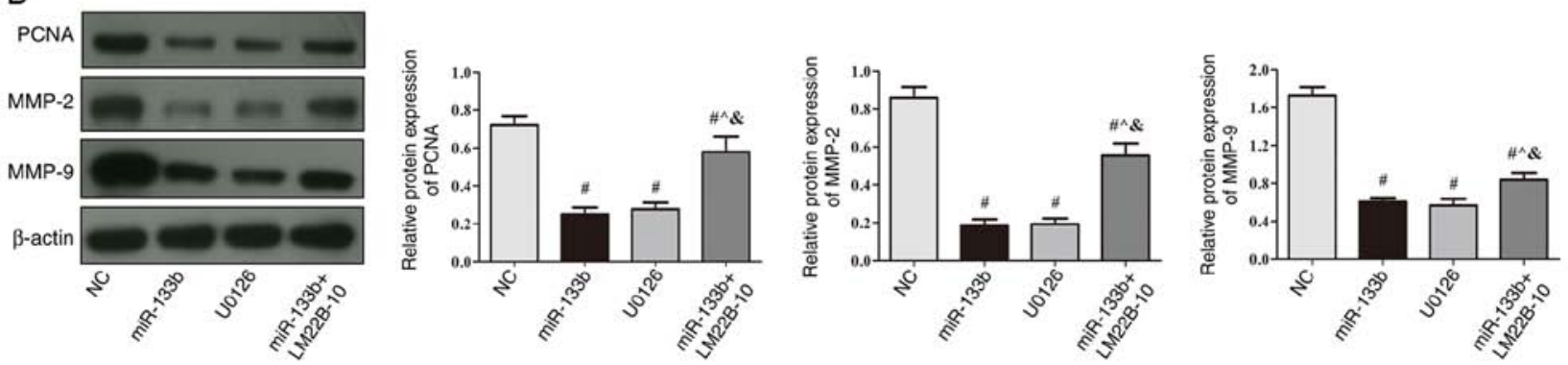

Figure 5. Overexpression of miR-133b inhibits 786-O cell proliferation and invasion by inhibiting the ERK signaling pathway. (A) Cell proliferation was determined in cells treated with U0126 (ERK pathway signal transduction inhibitor) or transfected with miR-133b mimic with or without LM22B-10 (ERK activator) treatment using an MTT assay. (B) Matrigel assays were used to determine the number of invasive cells in cells treated with U0126 or transfected with miR-133b mimic with or without LM22B-10 treatment, scale bar, $50 \mu \mathrm{m}$. (C) Wound healing assays in cells treated with U0126 or transfected with miR-133b mimic with or without LM22B-10 treatment were used to detect cellular migration, scale bar, $200 \mu \mathrm{m}$. (D) Western blotting was used to detect PCNA, MMP-2 and MMP-9 protein expression levels in cells treated with U0126 or transfected with miR-133b mimic with or without LM22B-10 treatment. ${ }^{\#} \mathrm{P}<0.05$ vs. NC; ${ }^{\wedge} \mathrm{P}<0.05$ vs. miR-133b; ${ }^{\&} \mathrm{P}<0.05$ vs. U0126. miR, microRNA; NC, negative control; OD, optical density; PCNA, proliferating cell nuclear antigen; MMP, matrix metalloproteinase.

But compared with the miR-133b group or U0126 group, the expression of p-ERK1/2/ERK1/2 was significantly increased in miR-133b + LM22B-10 group $(\mathrm{P}<0.05)$. These results suggested that the overexpression of miR-133b combined with LM22B-10 significantly reversed or weakened the anticancer effects of miR-133b overexpression.

\section{Discussion}

Due to the current lack of distinct symptoms and signs in early stage renal cell carcinoma, the majority of clinical cases present with middle-to-late stage cancer upon diagnosis; unfortunately, the therapeutic treatment of advanced 

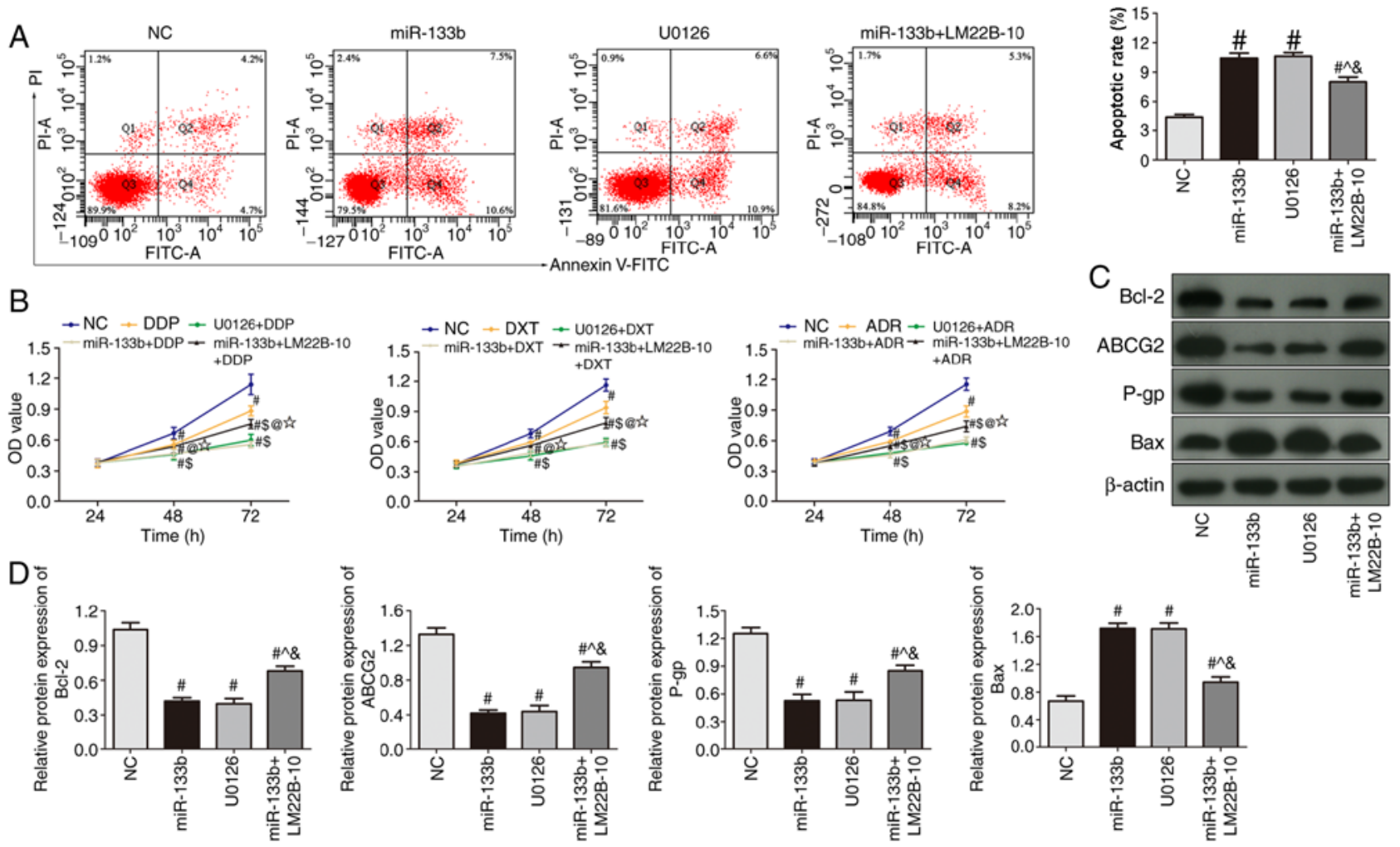

Figure 6. Overexpression of miR-133b induces the apoptosis of 786-O cells and enhances drug sensitivity through inhibiting the ERK signaling pathway. (A) Flow cytometric analysis of apoptosis in cells treated with U0126 or transfected with miR-133b mimic with or without LM22B-10 treatment. (B) Changes in the proliferation rate of 786-O cells treated with DDP, DXT or ADR alone, or in combination with U0126 or miR-133b overexpression with or without LM22B-10 treatment. (C) Western blotting was used to detect the protein expression levels of Bcl-2, Bax, ABCG2 and P-gp in cells treated with U0126 or transfected with miR-133b mimic with or without LM22B-10 treatment. (D) Semi-quantitative analysis of protein expression levels. ${ }^{\#} \mathrm{P}<0.05$ vs. NC; ${ }^{\wedge} \mathrm{P}<0.05$ vs. miR-133b; ${ }^{\circledR} \mathrm{P}<0.05$ vs. U0126; ${ }^{\$} \mathrm{P}<0.05$ vs. DDP/DXT/ADR; ${ }^{\circledR} \mathrm{P}<0.05$ vs. miR-133b group + DDP/DXT/ADR; ${ }^{\star} \mathrm{P}<0.05$ vs. U0126 + DDP/DXT/ADR. miR, microRNA; DDP, cisplatin; DXT, docetaxel; ADR, doxorubicin; UO126, ERK pathway signal transduction inhibitor; LM22B-10, ERK activator; NC, negative control; PI, propidium iodide; ABCG2, ATP-binding cassette subfamily G2; P-gp, p-glycoprotein; OD, optical density.

A

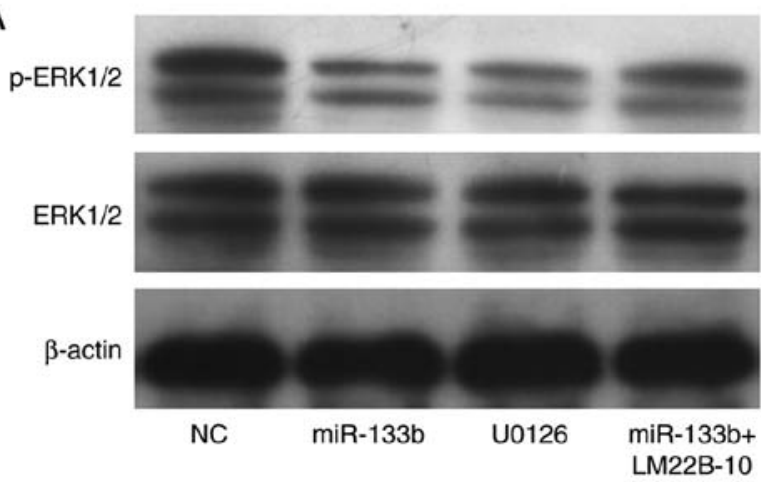

B

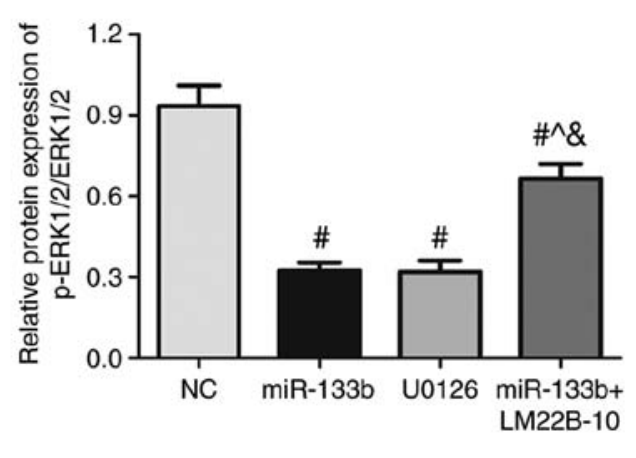

Figure 7. miR-133b overexpression inhibits ERK signaling pathway activity. (A) Western blotting was performed to investigate the protein expression levels of p-ERK1/2 and ERK1/2 in cells treated with the ERK inhibitor U0126, or transfected with miR-133b mimic with or without LM22B-10 (ERK activator) treatment. (B) Semi-quantification of the expression levels. ${ }^{*} \mathrm{P}<0.05$ vs. NC; ${ }^{\wedge} \mathrm{P}<0.05$ vs. miR-133b; ${ }^{\circledR} \mathrm{P}<0.05$ vs. U0126. miR, microRNA; NC, negative control; p, phosphorylated.

renal cell carcinoma is poor and the survival rate is low (17). Surgical resection remains an effective treatment option for local, early stage renal cell carcinoma; however, $>1 / 3$ of patients will eventually develop metastatic disease (2). Immunotherapy has also been observed to moderately prolong the survival time of patients in circumstances when chemotherapy and radiation therapy are not effective, and it has even been found to promote tumor regression and long-term survival in a small number of patients (18). Therefore, it is an urgent requirement to identify appropriate tumor biomarkers for the early diagnosis, treatment and prognosis of renal cell carcinoma (19). The present study found miR-133b expression in renal cell carcinoma was significantly lower compared with adjacent healthy tissues. Moreover, miR-133b expression in four types of renal cancer cells was lower compare with human embryonic kidney cells. 
miRNAs are abnormally expressed in all cancer cells, and it has been reported that this abnormal expression can regulate the activity of signaling pathways in cancer cells (7). Thus, the regulation of miRNAs over target genes may prevent certain diseases in humans. Conversely, changes in the expression levels of miRNAs has also been reported to lead to cancer occurrence (20); therefore, investigating the expression levels of miRNAs in tumor tissues may provide a theoretical basis for an improved understanding of cancer types (20). In the present study, the overexpression of miR-133b significantly decreased cell proliferation, and the migratory and invasive ability of 786-O cells, whilst increasing the rate of apoptosis. Concurrently, the protein expression levels of PCNA, MMP-2 and MMP-9, which are related to cell proliferation and invasion, were significantly decreased (11). These results preliminarily suggested a potential inhibitory effect of miR-133b in renal cell carcinoma.

$\mathrm{ABCG} 2$ is a member of the $\mathrm{ABC}$ transporter family; it has been found that $\mathrm{ABCG} 2$ can affect the concentration of intracellular chemotherapeutic drugs, in addition to inhibiting the activity of certain chemotherapeutic drugs or affecting their metabolites, which overall results in a poor response to chemotherapeutic regimens (21). Similar to P-gp, ABCG2 can actively pump chemotherapeutic drugs with different chemical structures and target sites out of cells, which subsequently induces tumor resistance to various anticancer drugs and reduces the sensitivity of cancer cells to chemotherapeutic drugs $(22,23)$. To investigate the effect of miR-133b on chemosensitivity in renal cell carcinoma, miR-133b was overexpressed and three chemotherapeutic drugs, DDP, DXT and ADR, were used to treat the cells. The results indicated that miR-133b overexpression increased the sensitivity of the 786-O cell line to these drugs. In addition, overexpression of miR-133b could decrease the expression levels of ABCG2 and P-gp. Concurrently, miR-133b overexpression reduced the expression levels of the anti-apoptotic protein, Bcl-2, and increased the expression levels of the pro-apoptotic protein, Bax. These findings further suggested that the anticancer effect of miR-133b may be related to the induced enhanced chemosensitivity of renal cell carcinoma cells and the increased apoptotic rate of the cancer cells.

The activation of ERK by phosphorylation is a prerequisite for the function of the ERK signaling pathway $(14,15)$. The present results demonstrated that the phosphorylation level of ERK was significantly reduced following the treatment with U0126 or miR-133b mimic. Bcl-2 and Bax are proteins involved in mitochondrial apoptosis (24), and P-gp, MMP-2 and MMP-9 are mitogen-activated protein kinase/ERK signaling pathway-related factors $(24,25)$. The present study observed that U0126 or miR-133b mimic treatment could also increase the protein expression levels of Bax and decrease the protein expression levels of MMP-2, MMP-9, P-gp and Bcl-2. However, the overexpression of miR-133b combined with LM22B-10 treatment was discovered to reverse or weaken the anticancer effects of miR-133b overexpression, which suggested that miR-133b may serve a role as a tumor suppressor gene through mediating the expression levels of ERK in renal cell carcinoma.

In conclusion, the findings from the present study indicated that miR-133b is expressed at low levels in renal cell carcinoma tissues and cells. The overexpression of miR-133b was found to inhibit the proliferation, migration and invasion of renal cell carcinoma cells, in addition to inducing apoptosis and enhancing the sensitivity of renal cell carcinoma cells to chemotherapeutic drugs; these functions may be related to the induction of ERK1/2 dephosphorylation, and thus the inhibition of the ERK signaling pathway. Since ERK is the convergence point of multiple signaling pathways, whether miR-133b affects the expression of genes upstream of ERK requires further study.

\section{Acknowledgements}

Not applicable.

\section{Funding}

No funding was received.

\section{Availability of data and materials}

The datasets used and/or analyzed during the current study are available from the corresponding author on reasonable request.

\section{Authors' contributions}

XY designed the study and drafted the manuscript. YM and XLL performed the experiments. XLL and SLG performed the statistical analysis. All authors read and approved the final manuscript.

\section{Ethics approval and consent to participate}

The present study was approved by the Research Ethics Committee of School of Medicine, Shandong University and was conducted under the Declaration of Helsinki principles. Informed written consent was obtained from all participating patients.

\section{Patient consent for publication}

Not applicable.

\section{Competing interests}

The authors declare that they have no competing interests.

\section{References}

1. White NM and Yousef GM: MicroRNAs: Exploring a new dimension in the pathogenesis of kidney cancer. BMC Med 8: 65, 2010.

2. Siegel RL, Miller KD and Jemal A: Cancer statistics, 2018. CA Cancer J Clin 68: 7-30, 2018.

3. Chen C, Xue S, Zhang J, Chen W, Gong D, Zheng J, Ma J, Xue W, Chen Y,Zhai W and Zheng J: DNA-methylation-mediated repression of miR-766-3p promotes cell proliferation via targeting SF2 expression in renal cell carcinoma. Int J Cancer 141: 1867-1878, 2017.

4. Zhai W, Li S, Zhang J, Chen Y, Ma J, Kong W, Gong D, Zheng J, Xue W and Xu Y: Sunitinib-suppressed miR-452-5p facilitates renal cancer cell invasion and metastasis through modulating SMAD4/SMAD7 signals. Mol Cancer 17: 157, 2018. 
5. Yan L, Ding B, Liu H, Zhang Y, Zeng J, Hu J, Yao W, Yu G, An $\mathrm{R}$, Chen Z, et al: Inhibition of SMYD2 suppresses tumor progression by down-regulating microRNA-125b and attenuates multi-drug resistance in renal cell carcinoma. Theranostics 9: 8377-8391, 2019.

6. Ambros V: The functions of animal microRNAs. Nature 431: 350-355, 2004

7. Bartel DP: MicroRNAs: Genomics, biogenesis, mechanism, and function. Cell 116: 281-297, 2004.

8. Palanichamy JK and Rao DS: miRNA dysregulation in cancer: Towards a mechanistic understanding. Front Genet 5: 54, 2004.

9. Juan D, Alexe G, Antes T, Liu H, Madabhushi A, Delisi C, Ganesan S, Bhanot G and Liou LS: Identification of a microRNA panel for clear-cell kidney cancer. Urology 75: 835-841, 2010.

10. Redova M, Poprach A, Nekvindova J, Iliev R, Radova L, Lakomy R, Svoboda M, Vyzula R and Slaby O: Circulating miR-378and miR-451 in serum are potential biomarkers for renal cell carcinoma. J Transl Med 10: 55, 2012.

11. Zhen Y, Liu J, Huang Y, Wang Y, Li W and Wu J: miR-133b inhibits cell growth, migration, and invasion by targeting MMP9 in non-small cell lung cancer. Oncol Res 25: 1109-1116, 2017.

12. Zeng W, Zhu JF, Liu JY, Li YL, Dong X, Huang H and Shan L: miR-133b inhibits cell proliferation, migration and invasion of esophageal squamous cell carcinoma by targeting EGFR Biomed Pharmacother 111: 476-484, 2019.

13. Roskoski R Jr: ERK1/2 MAP kinases: Structure, function, and regulation. Pharmacol Res 66: 105-143, 2012.

14. Qin W, Dong P, Ma C, Mitchelson K, Deng T, Zhang L, Sun Y, Feng X, Ding Y, Lu X, et al: MicroRNA-133b is a key promoter of cervical carcinoma development through the activation of the ERK and AKT1 pathways. Oncogene 31: 4067-4075, 2012.

15. Li S, Cui Z and Meng X: Knockdown of PARP-1 inhibits proliferation and ERK signals, increasing drug sensitivity in osteosarcoma U2OS cells. Oncol Res 24: 279-286, 2016.

16. Livak JK and Schmittgen TD: Analysis of relative gene expression data using quantitative PCR and the 2(-Delta Delta C(T)) method. Methods 25: 402-408, 2001.

17. Rasmussen F: Metastatic renal cell cancer. Cancer Imaging 13: 374-380, 2013.
18. Gardner CS, Ensor JE, Ahrar K, Huang SY, Sabir SH, Tannir NM, Lewis VO and Tam AL: Cryoablation of bone metastases from renal cell carcinoma for local tumor control. J Bone Joint Surg Am 99: 1916-1926, 2017.

19. Romero-Laorden N, Doger B, Hernandez M, Hernandez C, Rodriguez-Moreno JF and Garcia-Donas J: Predictive biomarker candidates to delineate efficacy of antiangiogenic treatment in renal cell carcinoma. Clin Transl Oncol 18: 1-8, 2016.

20. Rupaimoole R, Calin GA, Lopez-Berestein G and Sood AK: miRNA deregulation in cancer cells and the tumor microenvironment. Cancer Discov 6: 235-246, 2016.

21. Bram EE, Stark M, Raz S and Assaraf YG: Chemotherapeutic drug-induced ABCG2 promoter demethylation as a novel mechanism of acquired multidrug resistance. Neoplasia 11: 1359-1370, 2009.

22. To KK, Poon DC, Wei Y, Wang F, Lin G and Fu LW: Vatalanib sensitizes $\mathrm{ABCB} 1$ and $\mathrm{ABCG}$-overexpressing multidrug resistant colon cancer cells to chemotherapy under hypoxia. Biochem Pharmacol 97: 27-37, 2015.

23. Reustle A,FiselP,RennerO,BüttnerF,WinterS, RauschS,KruckS, Nies AT, Hennenlotter J, Scharpf M, et al: Characterization of the breast cancer resistance protein (BCRP/ABCG2) in clear cell renal cell carcinoma. Int J Cancer 143: 3181-3193, 2018.

24. Sun Y, Wang C, Meng Q, Liu Z, Huo X, Sun P, Sun H, Ma X, Peng $\mathrm{J}$ and Liu K: Targeting P-glycoprotein and SORCIN: Dihydromyricetin strengthens anti-proliferative efficiency of adriamycin via MAPK/ERK and $\mathrm{Ca}^{2+}$-mediated apoptosis pathways in MCF-7/ADR and K562/ADR. J Cell Physiol 233: 3066-3079, 2018.

25. Han CK, Tien YC, Jine-Yuan Hsieh D, Ho TJ, Lai CH, Yeh YL, HsuanDay C,Shen CY,HsuHH,Lin JY andHuang CY: Attenuation of the LPS-induced,ERK-mediated upregulation of fibrosis-related factors FGF-2, uPA, MMP-2, and MMP-9 by Carthamus tinctorius L in cardiomyoblasts. Environ Toxicol 32: 754-763, 2017.

This work is licensed under a Creative Commons Attribution-NonCommercial-NoDerivatives 4.0 International (CC BY-NC-ND 4.0) License. 